\title{
CARLEMAN'S FORMULAS AND CONDITIONS OF ANALYTIC EXTENDABILITY
}

\author{
L. A. AIZENBERG \\ Department of Mathematics, Bar-Ilam University \\ Ramat-Gan, 52 900, Israel
}

1. Criteria for analytic continuation into a domain of a function given on part of the boundary. The following classical assertion is well known. Let $\mathcal{D} \subset \mathbb{C}$ be a simply connected bounded domain with smooth boundary $\partial \mathcal{D}$ and $f \in C(\partial \mathcal{D})$. Then

$$
\int_{\partial \mathcal{D}} f z_{1}^{m} d z_{1}=0, \quad m=0,1,2, \ldots
$$

if and only if $f(z)$ extends into the domain $\mathcal{D}$ as a holomorphic function of class $A(\mathcal{D}) \cap C(\overline{\mathcal{D}})$. For the multidimensional case instead of the form $z_{1}^{m} d z_{1}$ we have the exterior differential form of class $Z_{n, n-1}^{\infty}(\bar{D})$.

Theorem 1 (Weinstock-Aronov-Dautov). Let $\mathcal{D}$ be a domain in $\mathbb{C}^{n}$ with smooth boundary and $f \in C(\partial \mathcal{D})$. Then there is a function $F \in A(\mathcal{D}) \cap C(\overline{\mathcal{D}})$ such that $\left.F\right|_{\partial \mathcal{D}}=f$ if and only if

$$
\int_{\partial \mathcal{D}} f \alpha=0
$$

for every form $\alpha \in Z_{n, n-1}^{\infty}(\overline{\mathcal{D}})$.

If $f$ is only defined on a part of the boundary of $\mathcal{D}$, then the existence of an analytic continuation into $\mathcal{D}$ cannot be decided by the vanishing of some family of continuous linear functionals (as in (1)-(2)). Solutions to this problem were given by G. Zin (1953), V. A. Fok-F. M. Kuni (1959), D. I. Patil (1972), M. G. Krein-P. Ya. Nudelman (1973), A. Steiner (1974), N. N. Tarkhanov (1989), O. V. Karepov-N. N. Tarkhanov (1990), A. A. Shlyapunov-N. N. Tarkhanov

1991 Mathematics Subject Classification: 32E35, 35R35, 32G07.

The paper is in final form and no version of it will be published elsewhere. 
(1990), L. Zhamenskaya (1990), L. A. Aizenberg (1988, 1990, 1991, 1992), L. A. Aizenberg-A. M. Kytmanov (1990, 1991), L. A. Aizenberg-C. Rea (1991). A very simple solution was given by L. A. Aizenberg (1990,1992):

Case 1: $n=1$. Let $\mathcal{D}$ be the domain bounded by a part of the unit circle $\gamma_{1}=\left\{z_{1}:\left|z_{1}\right|=1\right\}$ and a smooth open arc $\Gamma$ connecting two points of $\gamma_{1}$ and lying inside $\gamma_{1}$. Let $0 \notin \overline{\mathcal{D}}$. We set

$$
a_{k}=\int_{\Gamma} \frac{f(\zeta) d \zeta}{\zeta^{k+1}}, \quad k=0,1,2, \ldots
$$

THEOREM 2. If $f \in C(\Gamma) \cap L^{1}(\Gamma)$, then there is a function $F \in A(\mathcal{D}) \cap C(\mathcal{D} \cup \Gamma)$ such that $\left.F\right|_{\Gamma}=g$ if and only if

$$
\varlimsup_{k \rightarrow \infty} \sqrt[k]{\left|a_{k}\right|} \leq 1
$$

If $\left.f\right|_{\Gamma}$ is not identically zero, then (3) is equivalent to

$$
\lim _{k \rightarrow \infty} \sqrt[k]{\left|a_{k}\right|}=1
$$

Proof. Necessity. Put $\Gamma_{\varepsilon}=\left\{z_{1}:\left|z_{1}\right|<1-\varepsilon\right\} \cap \Gamma$, where $0<\varepsilon<1$, and

$$
a_{k}^{\varepsilon}=\int_{\Gamma_{\varepsilon}} \frac{f(\zeta) d \zeta}{\zeta^{k+1}} .
$$

Suppose there exists a function $F$ as in the Theorem. Then the $a_{k}^{\varepsilon}$ are equal to the corresponding integrals of $F / \zeta^{k+1}$ over the part of the circle $\gamma_{1-\varepsilon}$, therefore,

$$
\left|a_{k}^{\varepsilon}\right| \leq \frac{C(\varepsilon)}{(1-\varepsilon)^{k+1}}
$$

Also

$$
a_{k}=a_{k}^{\varepsilon}+\underset{\Gamma \backslash \Gamma_{\varepsilon}}{\int^{k+1}} \frac{f(\zeta) d \zeta}{\zeta^{k+1}}
$$

hence,

Now we obtain

$$
\left|a_{k}\right| \leq \frac{C(\varepsilon)}{(1-\varepsilon)^{k+1}}+\frac{C_{1}}{(1-\varepsilon)^{k+1}}
$$

$$
\varlimsup_{k \rightarrow \infty} \sqrt[k]{\left|a_{k}\right|} \leq \frac{1}{1-\varepsilon}
$$

whence we arrive at $(3)$ as $\varepsilon \rightarrow+0$.

Sufficiency. Consider the Cauchy type integral

$$
\frac{1}{2 \pi i} \int_{\Gamma} \frac{f(\zeta) d \zeta}{\zeta-z}=F_{ \pm}(z)
$$

which defines a function $F_{+}$holomorphic in $\mathcal{D}$ and a function $F_{-}$holomorphic in $\mathcal{D}_{-}$(part of the unit disc after the removal of $\overline{\mathcal{D}}$ ) such that the difference between 
their limit values along normals (points $z^{+}$and $z^{-}$equally distant from $\zeta$ ) on $\Gamma$ is equal to $f(\zeta)$ : for $\zeta \in \Gamma$,

$$
F_{+}(\zeta)-F_{-}(\zeta) \stackrel{\text { def }}{=} \lim _{z^{ \pm} \rightarrow \zeta}\left[F_{+}\left(z^{+}\right)-F_{-}\left(z^{-}\right)\right]=f(\zeta) .
$$

Moreover, if one of the functions $F_{+}$or $F_{-}$is continuous in the corresponding domain up to $\Gamma$, so is the other.

Expanding (5) into a power series in $z$ in a neighborhood of zero, we find that the coefficients of this series are $a_{k} /(2 \pi i)$. This and (3) imply that $F_{-}$is holomorphic on the whole unit disc. Then $F_{+}-F_{-} \in A(\mathcal{D}) \cap C(\mathcal{D} \cup \Gamma)$, and by (6) we can take $F=F_{+}-F_{-}$.

If $\overline{\lim }_{k \rightarrow \infty} \sqrt[k]{\left|a_{k}\right|}<1$, then (5) is holomorphic in the disc with radius $R>1$ and the singularity $\Gamma$ is removable, hence the second part of Theorem 2 is true.

Corollary 1. Let $f \in C(\Gamma)$. There is a function $F \in A(\mathcal{D}) \cap C(\mathcal{D} \cup \Gamma)$ such that $\left.F\right|_{\Gamma}=f$ if and only if

$$
\varlimsup_{k \rightarrow \infty} \sqrt[k]{\left|a_{k}^{\varepsilon}\right|} \leq \frac{1}{1-\varepsilon} \quad \text { for } 0<\varepsilon<\varepsilon_{0}<1 .
$$

Let us generalize this result to a simply connected domain $\Omega$ with Jordan boundary $\partial \Omega$ containing a smooth open arc $\Gamma$. We connect the ends of $\Gamma$ by a curve $\mathcal{C}$, lying outside $\bar{\Omega}$, and let $\Omega_{1}$ be the domain with boundary $\mathcal{C} \cup(\partial \Omega \backslash \Gamma)$, which we also assume to be Jordan. Let $w=\varphi\left(z_{1}\right)$ map $\Omega_{1}$ conformally onto the unit disc so that the preimage of zero is in $\Omega_{1} \backslash \bar{\Omega}$. Let

$$
A_{k}^{\varepsilon}=\int_{\Gamma_{\varepsilon}} \frac{f(\zeta) d \varphi(\zeta)}{\varphi^{k+1}(\zeta)} .
$$

Corollary 2. If $f \in C(\Gamma)$, then there is a function $F \in A(\Omega) \cap C(\Omega \cup \Gamma)$ such that $\left.F\right|_{\Gamma}=f$ if and only if

$$
\varlimsup_{k \rightarrow \infty} \sqrt[k]{\left|A_{k}^{\varepsilon}\right|} \leq \frac{1}{1-\varepsilon} \quad \text { for } 0<\varepsilon<\varepsilon_{0}<1 .
$$

We can apply the approach of this section to the case when $\Gamma=\partial \mathcal{D}$. Suppose 0 lies outside $\overline{\mathcal{D}}$, where $\mathcal{D}$ is a simply connected bounded domain with a smooth boundary. Then the classical condition for $f \in C(\partial \mathcal{D})$ to analytically continue to $\mathcal{D}$ is $(1)$.

COROLlaRY 3. The (necessary and sufficient) condition for analytic continuation of $f \in C(\partial \mathcal{D})$ to $\mathcal{D}$ is

$$
\varlimsup_{k \rightarrow \infty} \sqrt[k]{\left|\int_{\partial \mathcal{D}} f z_{1}^{k} d z_{1}\right|}<\rho=\min _{z \in \mathcal{D}}|z| .
$$

If (8) is true, then (1) is true. If the integrals in (1) do not grow too rapidly, they vanish. 
COROLlary 4. Given a simply connected domain $\Omega$ with Jordan boundary and a smooth open arc $\Gamma \subset \Omega, \Omega=\Omega_{1} \cup \Omega_{2} \cup \Gamma, \Omega_{1}$ and $\Omega_{2}$ simply connected domains. Let $f \in C(\Gamma)$. There exists a function $F \in A(\Omega)$ so that $\left.F\right|_{\Gamma}=f$ (this is not a boundary problem, but an interior problem) if and only if two conditions of type (7) hold.

EXAMPLE. Let $\Omega$ be the whole complex plane $\mathbb{C}$. In this case, a conformal mapping of $\mathcal{D}$ onto the unit disc does not exist, of course, but we do not need it. Let $\Gamma$ be a simply smooth curve, dividing the plane $\mathbb{C}$ into two domains: $\Omega_{1}$ and $\Omega_{2}$. Let $i \in \Omega_{1}$ and $-i \in \Omega_{2}$, and let

$$
a_{k}^{ \pm}=\int_{\Gamma} \frac{f(\zeta) d \zeta}{(\zeta \pm i)^{k+1}}, \quad k=0,1,2, \ldots,
$$

where $f \in C(\Gamma) \cap L^{1}(\Gamma)$. Then the function $f$ can be extended to an entire function if and only if

$$
\lim _{k \rightarrow \infty} \sqrt[k]{\left|a_{k}^{+}\right|}=\lim _{k \rightarrow \infty} \sqrt[k]{\left|a_{k}^{-}\right|}=0
$$

Case 2: $n>1$. Let $\Omega=\{\zeta: \psi(\zeta)<0\}$ be a $\left(p_{1}, \ldots, p_{n}\right)$-circular domain in $\mathbb{C}^{n}$, where $p_{1}, \ldots, p_{n}$ are natural numbers, i.e., $z \in \Omega$ implies $\left(z_{1}, e^{i t p_{1}}, \ldots, z_{n} e^{i t p_{n}}\right)$ $\in \Omega$ for $t \in \mathbb{R}$. In particular, for $p_{1}=\ldots=p_{n}=1$ this circular domain is a Cartan domain. Moreover, assume that $\Omega$ is convex and bounded and $\partial \Omega \in C^{2}$. Furthermore, let $\mathcal{D}$ be a domain bounded by a part of $\partial \Omega$ and by a hypersurface $\Gamma \in C^{2}$ dividing $\Omega$ into two parts, the complement of $\overline{\mathcal{D}}$ containing the origin. Let us consider the Cauchy-Fantappiè differential form

$$
w(\zeta-z, w)=\frac{(n-1) !}{(2 \pi i)^{n}} \frac{\sum_{k=1}^{n}(-1)^{k-1} w_{k} d w[k] \wedge d \zeta}{\langle w, \zeta-z\rangle^{n}},
$$

where $d w[k]=d w_{1} \wedge \ldots \wedge d w_{k-1} \wedge d w_{k+1} \wedge \ldots \wedge d w_{n}, d \zeta=d \zeta_{1} \wedge \ldots \wedge d \zeta_{n},\langle a, b\rangle=$ $a_{1} b_{1}+\ldots+a_{n} b_{n}$; then $\operatorname{grad} \psi=\left(\partial \psi / \partial \zeta_{1}, \ldots, \partial \psi / \partial \zeta_{n}\right)$. By the Sard Theorem, $\operatorname{grad} \psi \neq 0$ for almost all $r$ on $\partial \Omega_{r}$, where $\Omega_{r}=r \Omega$ is a homothety of $\Omega$ with $0<r<1$. We will assume that $\operatorname{grad} \psi \neq 0$ on $\Gamma$. We set

$$
C_{q}=\frac{(|q|+n-1) !}{q !} \int_{\Gamma} f(\zeta)\left(\frac{\operatorname{grad} \psi}{\langle\operatorname{grad} \psi, \zeta\rangle}\right)^{q} \omega(\zeta, \operatorname{grad} \psi),
$$

where $q=\left(q_{1}, \ldots, q_{n}\right), q !=q_{1} ! \ldots q_{n} !,|q|=q_{1}+\ldots+q_{n}, w^{q}=w_{1}^{q_{1}} \ldots w_{n}^{q_{n}}$,

$$
a_{k}=\sum b_{q, s} C_{q} \bar{C}_{s}
$$

where

$$
b_{q, s}=\int_{\Omega} z^{q} \bar{z}^{s} d v
$$

$d v$ is the volume element in $\Omega$. We emphasize that the integral moments $C_{q}$ depend on $f$ and $\Gamma$, buth the moments $b_{q, s}$ depend only on $\Omega$. 
THEOREM 3. For a function $f \in C(\Gamma) \cap L^{1}(\Gamma)$ to have a holomorphic continuation $F \in A(\mathcal{D}) \cap C(\mathcal{D} \cup \Gamma)$ with $\left.F\right|_{\Gamma}=f$, it is necessary and sufficient that the following two conditions are fulfilled:

(i) $f$ is a $C R$ function on $\Gamma$,

(ii) $\lim _{k \rightarrow \infty} \sqrt[k]{a_{k}} \leq 1$.

We managed to find a very simple solution, using a complete system of holomorphic functions (weighted homogeneous polynomial of degree $k$ if it is homogeneous of degree $k$ with respect to $\left.z_{1}^{1 / p_{1}}, \ldots, z_{n}^{1 / p_{n}}\right)$. If we used the basis, the answer would not be so easy.

COROLlary 1: statement of Theorem 2.

Corollary 2 (Aizenberg-Kytmanov). Let $\Omega \subset \mathbb{C}^{n}$ be a bounded convex $n$ circular domain. Set $d_{q}(\Omega)=\max _{\bar{\Omega}}\left|z^{q}\right|$. For a function $f \in C(\Gamma) \cap L^{1}(\Gamma)$ to have a holomorphic continuation in $\mathcal{D}$ as above it is necessary and sufficient that

(i) $f$ is a $C R$ function on $\Gamma$,

(ii) $\varlimsup_{|q| \rightarrow \infty} \sqrt[|q|]{\left|C_{q}\right| d_{q}(\Omega)} \leq 1$.

2. Carleman formulas. Let $\mathcal{D}$ be a bounded domain in $\mathbb{C}^{n}$ with piecewise smooth boundary $\partial \mathcal{D}$ and let $M$ be a set of positive $(2 n-1)$-dimensional Lebesgue measure in $\partial \mathcal{D}$. We consider the following problem: if $f$ is a holomorphic function in $\mathcal{D}$ that is sufficiently well behaved at the boundary $\partial \mathcal{D}$, for example $f$ is continuous in $\overline{\mathcal{D}},\left(f \in A_{C}(\mathcal{D})\right)$, or $f$ is contained in the Hardy class $H^{1}(\mathcal{D})$, then how can it be reconstructed inside $\mathcal{D}$ by its values on $M$ with the help of an integral formula? The problem makes sense because $M$ is a uniqueness set for such functions (L. A. Aizenberg, 1959). Solutions to this problem were given by T. Carleman (1926), G. Goluzin-V. Krylov (1933), G. Zin (1953), V. FockF. Kuni (1959), D. Patil (1972), M. Krein-P. Nudelman (1973), L. AizenbergN. Tarkhanov (1988), A. Kytmanov-T. Nikitina (1989), L. Aizenberg (1984, 1985, 1990, 1991, 1992).

Three methods of solution are known, due to: 1) Carleman-Goluzin-Krylov, 2) Lavrent'ev, 3) Kytmanov. There is also a very general approach offered by N. Videnskiı̌-E. Gavurina-V. Khavin (1983) for $n=1$.

A very simple solution:

Case 1: $n=1$. If $M=\Gamma$ is an arc in the unit disc with ends on the unit circle, then we can give a simpler formula (see the beginning of example 3, sec. 1 in [1] and Goluzin-Krylov (1933))

$$
f(z)=\lim _{m \rightarrow \infty} \frac{1}{2 \pi i} \int_{\Gamma} f(\zeta)\left(\frac{z}{\zeta}\right)^{m} \frac{d \zeta}{\zeta-z} .
$$

We show that this simple formula can be easily obtained, not only by introducing a quenching function into the Cauchy formula (the Carleman-Goluzin-Krylov method), but also by approximating the Cauchy kernel on $\partial \mathcal{D} \backslash \Gamma$ (the Lavrent'ev 
method). This also yields simple Carleman formulas with holomorphic kernels, generalizing (1), in the multidimensional case. So, let $\mathcal{D}$ be a domain in the disc $U(0, r)$ whose boundary consists of an arc $\gamma \subset \partial U$ and a smooth arc $\Gamma$ lying in $U(0, r)$ and connecting the ends of $\gamma$, and let $0 \notin \overline{\mathcal{D}}$. By the Cauchy formula $(\gamma$ and $\Gamma$ oriented compatibly with $\partial \mathcal{D})$

$$
f(z)=\frac{1}{2 \pi i} \int_{\Gamma} \frac{f(\zeta) d \zeta}{\zeta-z}+\frac{1}{2 \pi i} \int_{\gamma} \frac{f(\zeta) d \zeta}{\zeta-z} .
$$

The kernel of the second term on the right-hand side of (2) has a series expansion

$$
\frac{1}{2 \pi i} \frac{1}{\zeta-z}=\frac{1}{2 \pi i} \sum_{k=0}^{\infty} \frac{z^{k}}{\zeta^{k+1}}
$$

which, for fixed $z \in \mathcal{D}$, converges uniformly on $\gamma$ with respect to $\zeta$. Therefore,

$$
\frac{1}{2 \pi i} \frac{1}{\zeta-z}=\lim _{m \rightarrow \infty} \frac{1}{2 \pi i} \sum_{k=0}^{m-1} \frac{z^{k}}{\zeta^{k+1}}=\lim _{m \rightarrow \infty} \frac{1}{2 \pi i} \frac{1-(z / \zeta)^{m}}{\zeta-z} .
$$

The last function under the limit is holomorphic with respect to $\zeta$ in $\overline{\mathcal{D}}$, hence

$$
0=\frac{1}{2 \pi i} \int_{\Gamma} f(\zeta) \frac{1-(z / \zeta)^{m}}{\zeta-z} d \zeta+\frac{1}{2 \pi i} \int_{\gamma} f(\zeta) \frac{1-(z / \zeta)^{m}}{\zeta-z} d \zeta
$$

Then we subtract (4) from (2) and pass to the limit as $m \rightarrow \infty$. Then the integral over $\gamma$ approaches zero by (3), and we have the formula (1).

Cas e 2: $n>1$. Let $\Omega$ be a circular convex bounded domain (Cartan domain) with boundary of class $C^{2}$ and let $\Gamma$ be a piecewise smooth hypersurface intersecting $\Omega$ and cutting from it the domain $\mathcal{D}, 0 \notin \overline{\mathcal{D}}$, i.e., the boundary $\partial \mathcal{D}$ is the union of $\Gamma$ and of a part of the boundary of $\Omega$, which we denote by $\gamma\left(\gamma \in C^{2}\right.$ is sufficient). Then there exists a Cauchy-Fantappiè formula for the domain $\mathcal{D}$ with kernel holomorphic in $z$. It can be constructed by using a "barrier" for $\gamma$ and a "barrier" for $\Gamma$, and the "glueing" of the barrier in the case where they do not match sufficiently smoothly at the joining of $\Gamma$ and $\gamma$ (cf. the proof of Theorems 12.1 and 12.3 in [1], method of Norguet) being passed over when integrating over some cycle in $\mathbb{C}^{2 n}$ lying on $\Gamma \cap \gamma$. I.e., in fact, integration will be over $\gamma, \Gamma$ and the faces $\gamma \cap \Gamma$. We write the last two cases conditionally as the integral over $\tilde{\Gamma}$ of some form $R(z, \zeta, d \zeta, d \bar{\zeta})$. Then, for $f \in A_{C}(\mathcal{D})$ and the points $z \in \mathcal{D}$,

$$
f(z)=\int_{\tilde{\Gamma}} f(\zeta) R(z, \zeta, d \zeta, d \bar{\zeta})+\int_{\gamma} f(\zeta) \omega(\zeta-z, \operatorname{grad} \rho),
$$

where $\Omega=\{\zeta: \rho(\zeta)<0\}, \rho \in C^{2}(\bar{\Omega})$.

A circular convex domain $\Omega$ is also linearly convex, i.e., the analytic tangent plane $\left\{z:\left\langle\rho^{\prime}(\zeta), \zeta-a\right\rangle=0\right\}$, where $\zeta \in \partial \Omega$, does not overlap $\Omega$. In other words, 
for $\zeta \in \partial \Omega$ and $z \in \Omega$ the inequality $\left\langle\rho^{\prime}(\zeta), \zeta-z\right\rangle \neq 0$ holds, or

$$
\frac{\left\langle\rho^{\prime}(\zeta), z\right\rangle}{\left\langle\rho^{\prime}(\zeta), \zeta\right\rangle} \neq 1
$$

Moreover, if $z \in \Omega$, then $z e^{i t} \in \Omega$, where $0 \leq t \leq 2 \pi$. Therefore, (6) implies that

$$
\left|\left\langle\frac{\rho^{\prime}(\zeta)}{\left\langle\rho^{\prime}(\zeta), \zeta\right\rangle}, z\right\rangle\right|<1, \quad z \in \Omega, \zeta \in \partial \Omega
$$

where $\rho^{\prime}(\zeta)=\operatorname{grad} \rho(\zeta)$.

Consequently, the kernel of the second integral in (5) has a series expansion for $z \in \mathcal{D}, \zeta \in \gamma$,

$$
\begin{gathered}
\omega\left(\zeta-z, \rho^{\prime}(\zeta)\right)=\frac{(n-1) !}{\left(2 \pi i\left\langle\rho^{\prime}, \zeta\right\rangle\right)^{n}} \sum_{k=0}^{\infty} \frac{(k+n-1) !}{k !(n-1) !}\left\langle\frac{\rho^{\prime}(\zeta)}{\left\langle\rho^{\prime}(\zeta), \zeta\right\rangle}, z\right\rangle^{k} \sigma, \\
\sigma=\sum_{j=1}^{n}(-1)^{j-1} \rho^{\prime} d \rho^{\prime}[j] \wedge d \zeta,
\end{gathered}
$$

which uniformly converges with respect to $\zeta$ on $\gamma$ for fixed $z \in \mathcal{D}$.

Let the function $\rho$ defining the domain $\Omega$ be such that every domain $\Omega(r)=$ $\{z: \rho(\zeta)<r\}$ is also convex, $0<r<1$, while $\min \rho$ is attained at the point 0 . Then, everywhere in $\bar{\Omega} \backslash\{0\}$ the inequality $\left\langle\rho^{\prime}(\zeta), \zeta\right\rangle \neq 0$ holds, and the form

$$
\varphi_{k}=\frac{1}{\left\langle\rho^{\prime}, \zeta\right\rangle^{n}}\left\langle\frac{\rho^{\prime}(\zeta)}{\left\langle\rho^{\prime},(\zeta), \zeta\right\rangle}, z\right\rangle^{k} \sigma
$$

is of class $Z_{n, n-1}^{1}(\mathcal{D})$ for every $k$. Hence, this form is orthogonal to the holomorphic functions when integrating over $\partial \Omega$ :

$$
0=\int_{\Gamma} f(\zeta) \sum_{k=0}^{m} \frac{(k+n-1) !}{k !} \varphi_{k}+\int_{\gamma} f(\zeta) \sum_{k=0}^{m} \frac{(k+n-1) !}{k !} \varphi_{k} .
$$

We subtract the equality (8) from (5) and pass to the limit as $m \rightarrow \infty$. Then the second integral in the obtained equality approaches zero, and we obtain the following assertion:

THEOREM 4. If $\gamma$ is a part of the boundary of a circular convex bounded domain $\Omega, \Gamma$ is a piecewise smooth hypersurface intersecting $\Omega$, and $\mathcal{D}$ is the domain with boundary $\partial \mathcal{D}=\gamma \cup \Gamma$, and $0 \notin \overline{\mathcal{D}}$, then for every function $f \in A_{C}(\mathcal{D})$ and $z \in \mathcal{D}$, the following Carleman formula with holomorphic kernel is valid:

(9) $f(z)=\int_{\tilde{\Gamma}} f(\zeta) R(z, \zeta, d \zeta, d \bar{\zeta})-\lim _{m \rightarrow \infty} \int_{\Gamma} f(\zeta) \frac{1}{(2 \pi i)^{n}} \sum_{k=0}^{m} \frac{(k+n-1) !}{k !} \varphi_{k}$,

where the $\varphi_{k}$ are given by the equality (7). 
We note that if $n=1, \rho(\zeta)=|\zeta|^{2}-r$, then $\rho^{\prime}(\zeta)=\bar{\zeta}$ and

$$
\left\langle\frac{\rho^{\prime}(\zeta)}{\left\langle\rho^{\prime},(\zeta), \zeta\right\rangle}, z\right\rangle=\frac{z}{\zeta}
$$

and from (9) we obtain (1). So, (9) is a direct generalization of (1) to the multidimensional case. Each differential form $\varphi_{k}$ is a homogeneous polynomial of degree $k$ in $z$.

COROLlaRY 1. If there exists a vector-valued function (a "barrier") $w=$ $w(z, \zeta), z \in \mathcal{D}, \zeta \in \Gamma$, such that $\langle w, \zeta-z\rangle \neq 0, w \in C_{\zeta}^{1}(\Gamma)$, and $w$ smoothly extends to $\rho^{\prime}$ on $\gamma \cap \Gamma$, then

$$
f(z)=\lim _{m \rightarrow \infty} \int_{\Gamma} f(\zeta)\left[\omega(\zeta-z, w)-\frac{1}{(2 \pi i)^{n}} \sum_{k=0}^{m} \frac{(k+n-1) !}{k !} \varphi_{k}\right] .
$$

Now let $\Omega$ be an $n$-circular domain (a Reinhardt domain). Then the series for $\omega\left(\zeta-z, \rho^{\prime}\right)$ with respect to a homogeneous polynomial in $z$ can be replaced by a power series in $z$ :

COROLlaRY 2. If under the conditions of Corollary $1, \gamma$ is part of the boundary of an $n$-circular convex bounded domain, then

$$
f(z)=\lim _{m \rightarrow \infty} \int_{\Gamma} f(\zeta)\left[\omega-\frac{1}{\left(2 \pi i\left\langle\rho^{\prime}, \zeta\right\rangle\right)^{n}} \sum_{|\alpha|=0}^{m} \frac{(|\alpha|+n-1) !}{\alpha !}\left(\frac{\rho^{\prime}(\zeta)}{\left\langle\rho^{\prime}, \zeta\right\rangle}\right)^{\alpha} z^{a} \sigma\right] .
$$

Corollary 3. If $\Omega=\{z:|z|<r\}$ is a ball, then

(10) $f(z)$

$$
=\lim _{m \rightarrow \infty} \int_{\Gamma} f(\zeta)\left[\omega-\frac{(n-1) !}{(2 \pi i)^{n}} \frac{\left(|\zeta|^{2 m}-\langle\bar{\zeta}, z\rangle^{m}\right)^{n}}{|\zeta|^{2 m n}\langle\bar{\zeta}, \zeta-z\rangle^{n}} \sum_{j=1}^{n}(-1)^{j-1} \bar{\zeta}_{j} d \bar{\zeta}[j] \wedge d \zeta\right] .
$$

Corollary 4. If $n=1, \Omega=U(0, r)$ is a disc, from formula (10) we obtain formula (1) again.

\section{References}

[1] L. A. Aizenberg, Carleman Formulas in Complex Analysis, Kluwer Academic Publishers, 1993. 